\title{
Nonlinear modeling of slewing flexible structures: ideal and non ideal approaches
}

\author{
André Fenili, Cayo Prado Fernandes Francisco, Karl Peter Burr, José Manoel Balthazar, \\ Reyolando Manoel Lopes Rebello da Fonseca Brasil \\ Universidade Federal do ABC (UFABC), Centro de Engenharia, Modelagem e Ciências Sociais \\ Aplicadas (CECS) / Aerospace Engineering Santo André, SP - Brazil
}

\begin{abstract}
The mathematical modelling of rotating nonlinear flexible beam-like wing, driven by a DC motor, with rectangular cross section is investigated here. The structure is mathematically modeled considering linear curvature and clamped-free boundary conditions. The flexible wing has an angle of attack which is considered constant. Nonlinearities resulting from the coupling between the angular velocity of the rotating axis and the transversal vibration of the beam are considered. A drag force and a lift force acting along the beam length are also included in the mathematical model. These forces are velocity dependent nonlinear excitations acting on the bean-like wing.
\end{abstract}

\section{Introduction}

Investigations about the dynamic behavior and control of nonlinear flexible beam-like structures represent an area of research of continuing interest to researchers and scientists around the world due to the wide range of application of such structures in different areas, such as aerospace, naval and oceanic engineering. The objectives of these studies are, in general, the design of more lightweight and faster structures for oceanic, aerospace and robotic applications. Recently, the world demands for green energy technologies also required the development of flexible structures, with new objectives and requirements, such as wind and oceanic energy platforms.

In dealing with these kind of rotating structures, the interaction between the angular displacement, $\theta$, also called slewing angle, and the flexible structure deflection, $v(\mathrm{x}, \mathrm{t})$, can be very important in some cases, as in high angular speed maneuvers [1-3]. The inclusion of the drag and lift effects, in this case, incorporates, although in a simple manner, the interaction between the structure and the surrounding fluid as, for example, air or water. This interaction plays an important role as the fluid dissipates the motion kinetic energy and executes work on the system that can significantly alter the control performance and efficiency. We will also include in the model the energy transference between the actuator and the flexible structure through an ideal and a non ideal system approach, where the actuator is given by a DC motor.

\section{The geometrical model}

The geometric model of the dynamic system investigated in this work is presented in Figure 1. 


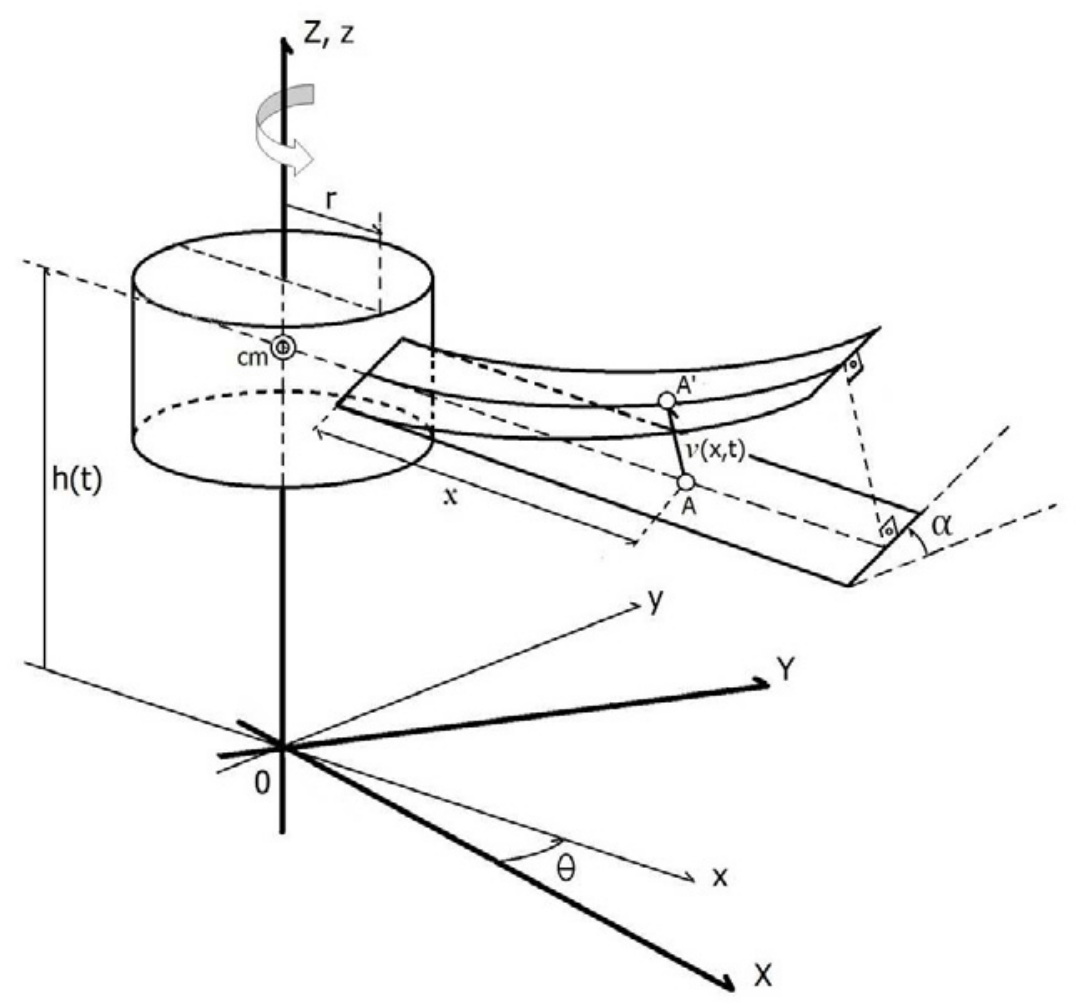

Figure 1. The rotating flexible beam system interacting with a fluid.

This system comprises a rigid hub, driven by a DC motor, and a flexible beam-like structure in rotation about $Z$ axis interacting with a quiescent fluid, where the effect on the motion of the beam is represented by the drag, $\mathrm{D}(\mathrm{x}, \mathrm{t})$ and lift, $\mathrm{L}(\mathrm{x}, \mathrm{t})$, forces, which are not represented on the figure.

The drag and lift forces, as considered in [4:], are functions of the velocities $\dot{v}(x, t)$ and $\theta(t)$. The lift is also a function of the vertical velocity $h(\mathrm{t})$. The model for the lift uses strip theory, and no tridimensional effects are included.

In this figure, the inertial axis is represented by XYZ, the moving axis (attached to the rotating axis and moving with it) is represented by xyz.

\section{The Mathematical Model}

We follow the approach developed in [4], which is shown here for the sake of clarity. The governing equations of motion are obtained through the energy method [5-9]. To apply this method one needs to know the kinetic, potential and strain energies stored in the hub and in the flexible structure during the time evolution.

The total kinetic energy, $T$, of the system is given by

$$
\begin{aligned}
& T=\frac{1}{2} m_{h u b} \dot{h}^{2}+\frac{1}{2} I_{h u b} \dot{\theta}^{2}+\frac{1}{2} \int_{0}^{\ell} \rho A\left[\dot{\theta}^{2}(\mathrm{r}+\mathrm{x})^{2}-2 \dot{\theta}(\mathrm{r}+\mathrm{x}) \dot{v} \sin \alpha+\dot{v}^{2}+\right. \\
& \left.+v^{2} \sin ^{2} \alpha \dot{\theta}^{2}+2 \dot{v} \cos \alpha \dot{h}+\dot{h}^{2}\right] d x
\end{aligned}
$$

In Equation (1), $\theta$ represents the angular displacement of the hub axis that is driven by an electrical DC motor, $h$ represents the vertical displacement of the hub, $r$ represents the radius of the hub, $\alpha$ represents the angle of attack of the beam, $v(\mathrm{x}, \mathrm{t})$ represents the transversal displacement of the beam, $\rho$ represents the density of the material that composes the beam, $A$ represents the beam cross section area and $l$ represents the non deflected length of the beam. 
In this model, linear curvature is assumed for the flexible structure [10-12]. The total potential energy, $V$, of the hub and of the rotating beam is given by

$$
V=m_{h u b} g h+\frac{1}{2} \int_{0}^{\ell} \rho A g(h+v \cos \alpha) d x+\frac{1}{2} \int_{0}^{\ell} E I v^{\prime \prime 2} d x
$$

where $E$ represents the Young's modulus of the material that composes the beam, and $I$ represents the moment of inertia of the cross-section area of the beam.

The Lagrangian, $L$, therefore, is given by

$$
\begin{aligned}
L= & \frac{1}{2} m_{h u b} \dot{h}^{2}+\frac{1}{2} I_{h u b} \dot{\theta}^{2}-m_{h u b} g h+\frac{1}{2} \int_{0}^{\ell} \rho A\left[\dot{\theta}^{2}(\mathrm{r}+\mathrm{x})^{2}-2 \dot{\theta}(\mathrm{r}+\mathrm{x}) \dot{v} \sin \alpha+\dot{v}^{2}+\right. \\
& \left.v^{2} \sin ^{2} \alpha \dot{\theta}^{2}+2 \dot{v} \cos \alpha \dot{h}+\dot{h}^{2}-2 g(h+v \cos \alpha)-\frac{E I}{\rho A} v^{\prime \prime 2}\right] d x
\end{aligned}
$$

The drag effect is inserted in Lagrange's equations as a generalized force, $\mathrm{D}$, that is given at any cross section by

$$
D_{\text {sec }}=\frac{1}{2} k U^{2}
$$

In Eq. (4), the proportionality constant $k$ is given by

$$
k=\rho_{f} S C_{D}
$$

with $\rho_{f}$ representing the density of the fluid, $S=c \sin \beta$, representing the section orthographic projection of the beam, $C_{D}$ representing the non dimensional drag coefficient and $\mathrm{c}$ is the beam cross sectional width (mean chord).

In the same manner one can also include the force component normal to the flow, called lift, as a generalized force.

Let a cross section of the beam be at a distance $\mathrm{x}$ from the hub. The lift generated by this beam section is given by

$$
L_{\text {sec }}=\frac{1}{2} \rho U^{2} c C_{L}(\beta)
$$

where $U$ is the modulus of the fluid velocity as seen by the beam cross section and $C_{L}$ is the lift coefficient for the beam cross section. $\mathrm{U}^{2}$ is given by

$$
U^{2}=[\dot{\theta}(r+x)-\dot{v} \sin \alpha]^{2}+[\dot{v} \cos \alpha+\dot{h}]^{2}
$$

and the angle $\beta$ is the angle of attack seen by the beam cross section. It is written as

$$
\beta(x, t)=\alpha-\tan ^{-1}\left(\frac{\dot{v} \cos \alpha+\dot{h}}{\dot{\theta}(r+x)-\dot{v} \sin \alpha}\right)
$$

and varies with the cross section position, $x$, along the beam and time. If one assumes that the horizontal velocity is much larger than the vertical velocity $(|\dot{\theta}(r+x)-\dot{v} \sin \alpha| \gg|\dot{v} \cos \alpha+\dot{h}|)$, then, the angle of attack is $\beta=\alpha$ and the sectional lift is in the vertical direction and the sectional drag is in the horizontal direction. This assumption simplifies the model. 
The virtual work generated by the cross sectional lift force and drag force is

$$
\delta W_{\text {sec }}=\left\{\vec{L}_{\text {sec }}+\vec{D}_{s e c}\right\} .\{v \sin \alpha \delta \theta \vec{\imath}+[(r+x) \delta \theta-\delta v \sin \alpha] \vec{\jmath}+[\delta v \cos \alpha+\delta h] \vec{k}\}
$$

With the assumption in the above paragraph, the sectional lift force is in the $z$ direction, while the drag force is in $y$ direction so that the virtual force due to the sectional lift and drag forces is given by

$$
\delta W_{s e c}=\frac{1}{2} \rho U^{2} c C_{L}(\alpha)[\delta v \cos \alpha+\delta h]-\frac{1}{2} k U^{2}[(r+x) \delta \theta-\delta v \sin \alpha]
$$

In order to obtain the total virtual work due to the lift and drag force along the beam, one needs to integrate the above expression along the beam. One obtains

$$
\begin{gathered}
\delta W_{f}=\frac{1}{2} \rho c \int_{0}^{l}\left\{[\dot{\theta}(r+x)-\dot{v} \sin \alpha]^{2}+[\dot{v} \cos \alpha+\dot{h}]^{2}\right\} C_{L}(\alpha)[\delta v \cos \alpha+\delta h] d x- \\
\frac{1}{2} k \int_{0}^{l}\left\{[\dot{\theta}(r+x)-\dot{v} \sin \alpha]^{2}+[\dot{v} \cos \alpha+\dot{h}]^{2}\right\}[(r+x) \delta \theta-\delta v \sin \alpha] d x .
\end{gathered}
$$

The extended Hamilton Principle can be written as

$$
\int_{t_{1}}^{t_{2}}[\delta \mathrm{W}+\delta \mathrm{L}] \mathrm{dt}=0
$$

In applying the extended Hamilton Principle we obtain the governing equations of motion for the variables $\mathrm{h}(\mathrm{t})$, and $v(\mathrm{x}, \mathrm{t})$ respectively, as given by [4]

$$
\begin{gathered}
\left(m_{h u b}+\rho A l\right) \ddot{h}+\rho A \cos \alpha \int_{0}^{l} \ddot{v} d x+\rho A g l-m_{h u b} g=F_{h} \\
\ddot{v}+(r+x) \sin \alpha \ddot{\theta}+\cos \alpha \ddot{h}-v \sin ^{2} \alpha \dot{\theta}^{2}+\rho A g \cos \alpha+\frac{E I}{\rho A} v^{i v}=f_{v}
\end{gathered}
$$

where $F_{h}$ and $f_{v}$ are given by

$$
\begin{gathered}
F_{h}=\frac{1}{2} \rho c \int_{0}^{l}\left\{[\dot{\theta}(r+x)-\dot{v} \sin \alpha]^{2}+[\dot{v} \cos \alpha+\dot{h}]^{2}\right\} C_{L}(\alpha) d x \\
f_{v}=\frac{1}{2} \rho c\left\{[\dot{\theta}(r+x)-\dot{v} \sin \alpha]^{2}+[\dot{v} \cos \alpha+\dot{h}]^{2}\right\}\left[C_{L}(\alpha) \cos \alpha+\frac{1}{2} k \sin \alpha\right]
\end{gathered}
$$

The equation of motion for the slewing angle, $\theta$, will be given by the mechanical energy equation governing the DC motor. The boundary conditions for the beam are given, as in [4], by

$$
\begin{aligned}
& v(0, t)=0 \\
& v^{\prime}(0, t)=0 \\
& v^{\prime \prime}(L, t)=0 \\
& v^{\prime \prime \prime}(L, t)=0
\end{aligned}
$$




\section{Ideal and Non ideal DC motor}

The electric tension balance in a DC motor controlled by its armor is obtained through Kirchoff law as

$$
L_{m} \frac{d i_{a}}{d t}+R_{a} i_{a}+K_{b} \dot{\theta}_{m}=U
$$

where $U$ represents the armor tension, $R_{d}$ is the armor resistance, $i_{a}$ the armor current, $L_{m}$ is the motor inductance, $\mathrm{K}_{\mathrm{b}}$ is the counter electromotive force constant and $\theta_{\mathrm{m}}$ represents the angular position of the motor.

The mechanical equation of the DC motor, controlled by armor, is given by the summation of the torques, relative to the motor axis, as

$$
\tau_{m}=K_{t} i_{a}-c_{m} \dot{\theta}_{m}-I_{m} \ddot{\theta}_{m}
$$

where $I_{m}$ is the inertia of the motor, $c_{m}$ represents the internal dissipation in the motor, $\tau_{\mathrm{m}}$ represents the torque applied on the mechanical transmission between the actuator and the moving structure and $\mathrm{K}_{\mathrm{t}}$ represents the motor torque constant.

We know that the angular displacement of the motor axis and the torque applied by the same axis to the transmission between the motor and the moving load are related to the angular displacement of the structure on which the motor is actuating and to the torque applied on the same structure by

$$
\begin{gathered}
\theta_{m}=N_{g} \theta \\
\tau=N_{g} \tau_{m}
\end{gathered}
$$

with $\tau$ representing the torque applied by the DC motor to the moving structure and $\mathrm{N}_{\mathrm{g}}$ the transmission relation between the motor and the structure. So we can write Eqs. (27) and (28) as

$$
\begin{gathered}
L_{m} \frac{d i_{a}}{d t}+R_{a} i_{a}+K_{b} N_{g} \dot{\theta}=U \\
I_{m} N_{g}^{2} \ddot{\theta}+c_{m} N_{g}^{2} \dot{\theta}-N_{g} K_{t} i_{a}+\tau=0
\end{gathered}
$$

The difference between the ideal and non-ideal dynamical system approximation is given by the choice of the actuator-structure coupling model, e.g., it depends on the way we model torque, $\tau$. For example, if the axis is sufficiently short, we can consider it as a rigid body, so that the total torque, $\tau$, acting on the structure can be written as

$$
\tau=-M+I_{\text {axis }} \ddot{\theta}
$$

so that $\mathrm{M}$ is the flexure moment of the beam, acting on the motor axis. In doing so, eq. (27) becomes

$$
\left(I_{\text {axis }}+I_{m} N_{g}^{2}\right) \ddot{\theta}+\left(c_{m} N_{g}^{2}\right) \dot{\theta}-\left(N_{g} K_{t}\right) i_{a}-M=0
$$

$\mathrm{M}$ can be represented by the linear curvature model, so that

$$
M=E I v^{\prime \prime} .
$$




\section{Ideal and non ideal system}

The equations of motion for the slewing flexible structure considering an ideal $(\beta=0)$ and non ideal $(\beta=1)$ power source are given by Eqs. (13) and (14) and by the motor equations

$$
\begin{gathered}
L_{m} \frac{d i_{a}}{d t}+R_{a} i_{a}+K_{b} N_{g} \dot{\theta}=U \\
\left(I_{\text {axis }}+I_{m} N_{g}^{2}\right) \ddot{\theta}+\left(c_{m} N_{g}^{2}\right) \dot{\theta}-\left(N_{g} K_{t}\right) i_{a}-\beta\left(E I v^{\prime \prime}\right)=0 .
\end{gathered}
$$

\section{Conclusions}

A mathematical model consisting of a flexible beam-like wing, driven by a DC motor, under the influence of aerodynamic drag and lift forces was derived yielding a deterministic system of coupled nonlinear equations for the description of its dynamics.

This system of equations, although carrying simplifying assumptions contains the fundamental physical processes of the flexible beam dynamics, considering its interaction with the actuator and the surrounding fluid, where drag and lift forces are modeled as generalized forces.

Our next step is to implement a nonlinear control method in order to eliminate the vibrations on the structure while controlling its vertical position and angular velocity.

\section{References}

[1] Telli, S., Kopmaz, O., "On The Mathematical Modelling Of Beams Rotating About a Fixed Axis", Mathematical and Computational Applications, Vol. 9, No. 3, pp. 333-347, 2004.

[2] Low, K. H., "Vibration Analysis of a Tip-Loaded Beam Attached to a Rotating Joint", Computers and Structures, vol. 52, no. 5, pp 955-968, 1994.

[3] Putter, S., Manor, H., "Natural Frequencies of Radial Rotating Beams", Journal of Sound and Vibrations", 56(2), pp 175-185, 1978.

[4] Fenili,A., Francisco, C.P.F., Burr, K.P., "Mathematical Modelling of a Rotating Nonlinear Flexible Beam-like Wing", Presentations of the International Congress on Vibration Problems (ICOVP), 2013.

[5] Meirovitch, L., “Analytical Methods in Vibrations", Macmillan Publishing Co., Inc., New York, 1967.

[6] Greenwood, D. T., “Classical Dynamics”, Dover Publications, Inc., New York, 1977.

[7] Fox, C., "An Introduction to the Calculus of Variations", Dover Publications, Inc., New York, 1987.

[8] Thomson, W. T., "Theory of Vibration with Applications”, Prentice Hall, New Jersey, 1988.

[9] Craig, Jr., R. R., "Structural Dynamics - An Introduction to Computer Methods", John Wiley and Sons, 1981.

[10]Fenili A., "Mathematical Modeling and Analysis of the Ideal and Nonideal Behavior of Slewing Flexible Structures", Ph.D Thesis, University of Campinas (UNICAMP), Faculty of Mechanical Engineering. Brazil, 2000. In Portuguese.

[11] Popov, E. P., "Introdução à Mecânica dos Sólidos", Editora Edgar Blücher Ltda, 1978.

[12] Sah, J. J., Mayne, R. W., "Modeling of a Slewing Motor-Beam System", Proceedings of the International Computers in Engineering Conference, Boston, pp 481-486, 1990. 